Revista de Psicología y Educación / Journal of Psychology and Education, 2021, 16(1), 31 -43 (www.rpye.es) Doi: https://doi.org/10.23923/rpye2021.01.200

ISSN: $1699-9517$

\title{
AVANCE ONLINE
}

\section{Capacidad predictiva del apoyo social percibido sobre el bienestar subjetivo del alumnado de Educación Secundaria}

\author{
Mercedes Nancy Jiménez-Rosario*, Igor Esnaola e Inge Axpe \\ Universidad del País Vasco/Euskal Herriko Unibertsitatea (UPV/EHU)
}

\begin{abstract}
Resumen: El objetivo del estudio consistió en analizar la capacidad predictiva de las diversas fuentes del apoyo social percibido (de personas significativas, de la familia y de las amistades) sobre el bienestar subjetivo (satisfacción con la vida, afecto positivo y afecto negativo) de los y las adolescentes considerando el sexo y la edad. La muestra estuvo compuesta por 1030 estudiantes con edades entre 11 y 19 años $(M=14,68$; DT $=1,73), 579$ chicas $(56,2 \%)$ y 451 chicos $(43,8 \%)$ residentes en la República Dominicana. Los cuestionarios utilizados fueron el Multidimensional Scale of Perceived Social Support (MSPSS) para medir el apoyo social percibido; y el Satisfaction with Life Scale (SWLS) y el Positive and Negative Affect Schedule (PANAS) para medir el bienestar subjetivo. Los resultados de este estudio muestran que las diferentes fuentes del apoyo social percibido son predictoras del bienestar subjetivo entre las chicas, mientras el apoyo de personas significativas y el apoyo familiar predicen el bienestar subjetivo entre los chicos. Estos resultados señalan el papel del sexo y la edad en la influencia del apoyo social percibido sobre el bienestar subjetivo en la etapa de la adolescencia.
\end{abstract}

Palabras clave: Apoyo de Personas Significativas, Apoyo Familiar, Apoyo de las Amistades, Bienestar Subjetivo.

\section{Predictive capacity of perceived social support on subjective well-being in students of Secondary Education}

Abstract: The objective of this study was to analyze the predictive capacity of the various sources of perceived
social support (significant persons, family members, and friendships) on subjective well-being (satisfaction
with life, positive affect and negative affect) of adolescents differentiating between gender and age. The
sample consisted on 1030 students aged between 11 and 19 years (M=14.68, SD 1.73 ), 579
girls (56.2\%) and 451 boys (43.8\%) living in the Dominican Republic. The questionnaires used were the
Multidimensional Scale of Perceived Social Support (MSPSS) to measure the perceived social support; and,
the Satisfaction with Life Scale (SWLS) and the Positive and Negative Affect Schedule (PANAS) to measure
the subjective well-being. The results of this study have indicated that the different sources of perceived
social support are predictors of subjective well-being in the female sample, and the support of significant
persons and family support predict subjective well-being in the male sample. These results point to the role
of gender and age in the influence of perceived social support on subjective well-being in the adolescent
stage.
Keywords: Support of Significant Persons, Family Support, Support from Friendships, Subjective Well-being.

La adolescencia es una época de grandes cambios (Wang y Zauszniewski, 2018) que ocurren de manera simultánea en todos los ámbitos vitales: físico, social, psicológico y cognitivo (Fuentes, García, Garcia y Alarcón, 2015; Hernando, Oliva y Pertegal, 2013).

Recibido: 29/09/2020 - Aceptado: 20/1 1/2020 - Avance online: 11/01/2021 *Correspondencia: Mercedes Nancy Jiménez Rosario.

Universidad del País Vasco/Euskal Herriko Unibertsitatea (UPV/ EHU)

Dirección: 20018, Gipuzkoa, España.

E-mail: mercedesnancyjimenez@gmail.com

Jiménez-Rosario, M. N. Esnaola, I. y Axpe, I. (2021). Capacidad predictiva del apoyo socia percibido sobre el bienestar subjetivo en el alumnado de educación secundaria. Revista de Psicología y Educación, 16(1), 31-43, hitps://doi.org/10.23923/rpye2021.01.200
Dicha etapa evolutiva se caracteriza por precisar de la resolución de una importante tarea evolutiva; la de la definición y construcción de la propia identidad (Orcasita y Uribe, 2012), de tal manera que, a menudo, los y las adolescentes se debaten entre la búsqueda de la independencia y la separación de la familia, y la necesidad de inclusión en grupos sociales (Cunsolo, 2017), lo que puede hacer que en esta etapa se vea en riesgo su nivel de bienestar (Ronen, Hamama,

1699-9517/@ 2021 Asociación Científica de Psicología y Educación (ACIPE). Publicado por Consejo General de Colegios Oficiales de Psicólogos, España. Este es un artículo Open Access bajo la CC BY-NC-ND licencia (http://creativecommons.org/licencias/bync-nd/4.0/. 
Rosenbaum y Mishely-Yarlap, 2016). Por esta razón, es importante que en este proceso transicional los y las adolescentes dispongan de recursos que les permitan afrontar los cambios de manera positiva. En este sentido, el apoyo social tiene una gran relevancia en el bienestar subjetivo de los adolescentes, ya que proporciona recursos valiosos para hacer frente a las diferentes situaciones de riesgo y problemas que se presentan en esta etapa evolutiva (González, Gaxiola y Venezuela, 2018; Vongsirimas, Phetrasuwan, Thanoi y Yobas, 2018).

El apoyo social puede definirse como las provisiones instrumentales o expresivas, reales o percibidas, procedentes de la sociedad o la comunidad, amistades íntimas y redes sociales que suceden en situaciones variadas, tanto cotidianas como de crisis (Lin, 1986). Se trata de un fenómeno o constructo multidimensional (Dambi et al., 2018; Vongsirimas et al., 2018) que incorpora elementos tanto tangibles como intangibles, pudiendo ser de tipo material, como de información (consejos), emocional (ofrecimiento de simpatía), o de pertenencia (afiliación con otros) (Bowen et al., 2014). Existen, por tanto, diversas formas de conceptualizar, operacionalizar y medir el apoyo social (Vongsirimas et al., 2018). No obstante, en este trabajo el énfasis se sitúa en el apoyo social subjetivo o percibido, aquel referido a la percepción subjetiva de que uno es amado y de que cuenta con personas a quienes recurrir en caso de necesidad (Barra, 2004). Entre las numerosas escalas existentes para la medida del apoyo social percibido, una de las más utilizadas, traducida y validada a múltiples idiomas (Dambi et al., 2018) es la Multidimensional Scale of Perceived Social Support (MSPSS de Zimet, Dahlem, Zimet y Farley, 1988). Dicha escala evalúa el apoyo social percibido de la familia y las amistades, incluyendo también el apoyo percibido de una tercera fuente definida como "persona especial o significativa", cuya interpretación queda a juicio de quien responde. Esto resulta relevante en la adolescencia, por ser un momento en el que se inician las relaciones de pareja y puede existir, también, una mayor influencia de personas adultas fuera de la familia (Vongsirimas et al., 2018). Así, todavía para los adolescentes, la primera fuente de apoyo social continúa siendo la familia, si bien la relación con iguales adquiere un peso importante en su bienestar (PalomarLever y Victorio-Estrada, 2014). De hecho, es frecuente que los y las adolescentes se identifiquen con sus iguales (amistades, pareja, etc.) para tratar de validar sus propios sentimientos y pensamientos, necesitando sentir la aceptación y aprobación de éstos (Orcasita y Uribe, 2012). Por ello, aunque la familia continúa ejerciendo un papel relevante en el desarrollo social y el bienestar subjetivo de los adolescentes (Alfaro, Casas y López, 2015; Rodríguez-Fernández, Ramos-Díaz, Fernández-Zabala y Revuelta, 2016), los y las jóvenes pasarán cada vez más tiempo con sus iguales (parejas, amistades), en busca de comprensión y aceptación social (Zavala, Valadez y Vargas, 2008), de tal manera que el apoyo percibido de dicha fuente va adquiriendo mayor importancia (Gaete, 2015).

El bienestar subjetivo es un concepto amplio y multifacético, al que a menudo se ha hecho referencia con distintas denominaciones, como bienestar psicológico, satisfacción vital, calidad de vida o felicidad, entre otros (Bisquerra, 2010).

Aunque existan distintas propuestas en relación a los componentes del bienestar subjetivo, destaca aquella que lo considera constituido por dos; uno emocional y otro cognitivo (Diener, Suh, Lucas y Smith, 1999). Desde esta perspectiva, el bienestar subjetivo constaría de dos componentes: (1) uno cognitivo (la satisfacción con la vida) y (2) otro de naturaleza afectiva denominado balance afectivo. A su vez, dentro de estos dos componentes se diferenciarían tres dimensiones, al distinguirse, dentro del componente afectivo, el afecto positivo y el negativo (Diener, 2009). En esta estructura tridimensional contrastada empíricamente (Albuquerque, de Lima, Matos y Figueiredo, 2012; Rodríguez-Fernández y GoñiGrandmontagne, 2011), la satisfacción con la vida se expresa en forma del juicio global que las personas hacen de su trayectoria vital 
(Diener, 1984), mientras que el nivel afectivo surge a modo de balance del conjunto de los afectos positivos y de los afectos negativos que marcan la vida de cada persona (Bradburn, 1969). Ambos componentes (cognición y afecto) conformarían sistemas diferentes: mientras la satisfacción con la vida es un compendio de toda la vida propia, el balance afectivo es el resultado de reacciones inmediatas y continuas a los eventos que nos suceden y constituirían dos dimensiones relativamente independientes, cuya incidencia sobre el bienestar subjetivo dependería de la frecuencia en que fuesen experimentados (Bradburn 1969).

Como se ha señalado, uno de los principales recursos con los que cuenta el o la adolescente es el apoyo social de su entorno cercano. Esta percepción de apoyo ha mostrado su relación e importancia con el bienestar subjetivo (Ronen et al., 2016; Zeidner, Matthews y Shemesh, 2016) y la satisfacción vital tanto entre adolescentes (Chen et al., 2017; Oberle, Schonert-Reichl y Zumbo, 2011 ) como entre jóvenes y adultos (Kong, Zhao y You, 2012; Liv, Li, Ling y Cai, 2016; Yang, Xia, Han y Liang, 2018). No obstante, ha sido frecuente que las investigaciones lo traten de manera global, sin diferenciar las fuentes de apoyo percibido.

Cuando se han tomado en cuenta las diversas fuentes y componentes del bienestar adolescente, el aspecto que, sin duda, se ha analizado con más frecuencia ha sido la satisfacción con la vida, y las fuentes más frecuentemente incluidas han sido el apoyo familiar y de las amistades. En este sentido, se ha constatado que el apoyo de la familia permite predecir la satisfacción con la vida, así como puntuaciones inferiores en afecto negativo (Rodríguez-Fernández et al., 2016) entre adolescentes españoles. El apoyo de las amistades también parece explicar, junto al de la familia, la satisfacción vital de adolescentes finlandeses y mexicanos (Haanpää, Kuula y Hakovirta, 2019; Palomar-Lever y Victorio-Estrada, 2014). Sin embargo, no existe consenso respecto a qué fuente es más importante. Cuando ambas se tienen en consideración, algunos estudios observan, entre adolescentes mexicanos y argentinos, que el apoyo familiar y el apoyo de las amistades predice de forma positiva la satisfacción con la vida y el afecto positivo, mientras que predice de manera negativa el afecto negativo (González, Torres y Morelaro, 2020). Otros estudios indican mayor relevancia del apoyo percibido de la familia (entre adolescentes brasileños y españoles) frente a las amistades (Sarriera, Bedin, Abs, Calza y Casas, 2015; Rodríguez-Fernández, Droguett y Revuelta, 2012) considerándolo, incluso, como el factor más importante en la percepción de satisfacción vital adolescente, tanto entre chicos como entre chicas españolas (González-Carrasco et al., 2017). Otras investigaciones, sin embargo, encuentran, en la adolescencia temprana con muestra española, una mayor relevancia de las amistades, si bien apuntan a que podría tratarse de un mecanismo compensatorio, ya que los adolescentes con menor satisfacción vital y peores relaciones familiares eran precisamente quienes conferían mayor importancia a las amistades (Navarro et al., 2017).

Así, parece que el apoyo percibido y el apego de amistades se han mostrado más relacionados con el componente afectivo del bienestar, observándose su capacidad predictiva sobre el afecto positivo (Balluerka, Gorostiaga, Alonso-Arbiol y Aritzeta, 2016; Rodríguez-Fernández et al., 2016) y negativo en el caso de los chicos adolescentes españoles (Azpiazu, Esnaola y Sarasa, 2016). Entre el alumnado de universidad, si bien en ciertos estudios y culturas como Turquía e Irán (Gülaçti, 2010; Matsuda, Tsuda, Kim y Deng, 2014) no se observa capacidad predictiva del apoyo de amistades sobre ningún componente del bienestar subjetivo, sí predice el afecto positivo entre estudiantes de Jordania y EE.UU (Brannan, Biswas-Diener, Mohr, Mortazavi y Stein, 2013), aunque en éste último caso el apoyo de la familia permite, igualmente, explicar todos los componentes del bienestar en todas las culturas. Otros trabajos también han encontrado dicha capacidad predictiva, pero dependiendo del sexo. Es decir, si bien el apoyo de la familia explicaba la satisfacción 
con la vida tanto entre chicos como entre chicas españolas a lo largo de toda la adolescencia, en lo relativo a los afectos mostraba capacidad predictiva del afecto positivo entre las chicas y del afecto negativo entre los chicos (Azpiazu et al., 2016). Otros estudios, utilizando el MSPSS con adolescentes peruanos, han constatado que el apoyo de la familia es la única fuente que permite explicar de manera significativa tanto menores puntuaciones de afecto negativo como positivo (Navarro-Loli, Merino-Soto, Domínguez-Lara y Lourenço, 2019).

En lo referente al apoyo de personas significativas, en muestra adulta se ha constatado que predice la satisfacción con la vida (Gallagher y Vella-Brodrick, 2008) así como el afecto positivo (Matsuda et al., 2014) y el negativo (Gallagher y VellaBrodrick, 2008), si bien en otros estudios este apoyo no se ha mostrado significativo (Gülaçti, 2010).

Es posible que, en parte, estas diferencias puedan deberse tanto al contexto o sociedad en la que se realiza el estudio (existiendo muy poca investigación aún en el contexto latinoamericano), como a la edad y al sexo de las personas participantes. De hecho, aunque la familia sigue siendo importante, algunos estudios observan cierto descenso de esta relevancia al final de la adolescencia media (Trejos-Herrera, Bahamón, AlarcónVásquez, Vélez y Vinaccia 2018), así como ciertas diferencias en función del sexo. En este sentido, se han constatado mayores puntuaciones en apoyo, como medida global, entre las chicas que entre los chicos (González et al., 2016), sin encontrar diferencias en relación al apoyo familiar (Sarriera et al., 2015), u obteniendo mayores puntuaciones los chicos (Norris y Ayres, 2016). Las chicas también parecen percibir mayor apoyo de amistades (Sarriera et al., 2015) así como de otras personas significativas (TrejosHerrera et al., 2018), además de mayor grado de intimidad y proximidad en sus relaciones de amistad (Barcelata, Granados y Ramírez, 2013; Barra, 2012). Parece, pues, que las chicas muestran más facilidad para establecer nuevos vínculos afectivos y percibir mayor apoyo de personas significativas que los chicos (Prabhu y Shekhar, 2017). Dadas estas diferencias encontradas en función del sexo en el apoyo social, así como la importancia que reviste conocer en qué medida un recurso externo como el apoyo social permite explicar el bienestar subjetivo adolescente, esta investigación tiene como objetivo general analizar la capacidad predictiva del apoyo social percibido sobre el bienestar subjetivo de estudiantes de secundaria. Los objetivos específicos son: 1) analizar la capacidad predictiva de tres fuentes del apoyo social percibido (de la familia, de las amistades y de personas significativas) sobre los componentes del bienestar subjetivo (satisfacción con la vida, afecto negativo y afecto positivo); 2) analizar la capacidad predictiva de tres fuentes del apoyo social percibido (de la familia, de las amistades y de personas significativas) sobre los componentes del bienestar subjetivo (satisfacción con la vida, afecto negativo y afecto positivo) tomando en cuenta el sexo; 3) analizar la capacidad predictiva de tres fuentes del apoyo social percibido (de la familia, de amistades y de personas significativas) sobre los componentes del bienestar subjetivo (satisfacción con la vida, afecto negativo y afecto positivo), en función de la edad y, por tanto, en función de la etapa de la adolescencia (temprana y media) en la que se encuentra el alumnado de secundaria. Se centra, además, en adolescentes de la República Dominicana, no conociéndose estudios previos que aborden esta temática en dicha población.

De estos objetivos derivan las siguientes hipótesis de trabajo: 1) el apoyo social percibido (de la familia, de las amistades y de personas significativas) predice el bienestar subjetivo de las y los adolescentes; 2) existen diferencias en la capacidad predictiva de las fuentes de apoyo social percibido sobre el bienestar subjetivo de los y las adolescentes en función del sexo; 3) existen diferencias en la capacidad predictiva de las fuentes de apoyo social percibido sobre el bienestar subjetivo de los y las adolescentes en función de la edad (adolescencia temprana o media). 


\section{MÉTODO}

\section{PARTICIPANTES}

En esta investigación participaron 1030 estudiantes de educación secundaria de ocho centros educativos (seis públicos y dos privados) con edades comprendidas entre los 11 y 19 años $(M=14,68$; DT $=1,73)$, de los cuales 579 eran chicas $(56,2 \%)$ y 451 eran chicos $(43,8 \%)$ residentes en la República Dominicana. La muestra se dividió en dos subgrupos, adolescencia temprana 11-14 años $(M=13,07 ; D T=, 900)$ y adolescencia media 15-19 años $(M=15,98 ; D T=, 973)$.

\section{INSTRUMENTOS}

Para medir el apoyo social se utilizó el cuestionario Multidimensional Scale of Perceived Social Support (MSPSS) de Zimet et al. (1988), traducido al español y validado en Chile por Arechavala y Miranda (2002). La escala MSPSS evalúa la percepción que tienen las personas del apoyo social informal con el que cuentan. Está compuesta por 12 ítems, los cuales recogen información del apoyo social percibido por los individuos en tres áreas: familia (por ej., "obtengo de mi familia la ayuda y el apoyo emocional que necesito"), amistades (por ej., "mis amigos realmente tratan de ayudarme") y otros significativos 0 personas significativas (por ej., "existe una persona especial con la cual yo puedo compartir penas y alegrías"). Los ítems se valoran empleando una escala de respuestas que oscila entre 1 = "totalmente en desacuerdo" y 7 = "totalmente de acuerdo", de manera que, a mayor puntuación, mayor es la percepción de apoyo social percibido. La consistencia interna en esta investigación en sus tres subescalas alcanza los siguientes valores: familia $(\alpha=, 80)$, amigos $(\alpha=84)$, y otros significativos $(\alpha=, 80)$.

Para evaluar el bienestar subjetivo se utilizaron dos cuestionarios. Uno de ellos es el Satisfaction with Life Scale (SWLS) de Diener, Emmons, Laser y Griffin (1985), validado al castellano por Atienza, Pons, Balaguer y García-Merita (2000). Se trata de una escala que evalúa la satisfacción con la vida de forma global, referida a los propósitos e ideales propuestos y conseguidos a través de 5 ítems: "En la mayoría de las cosas, mi vida está cerca de mi ideal", "Las condiciones de mi vida son excelentes", "Estoy satisfecho con mi vida", "Hasta ahora, he conseguido las cosas que para mí son importantes en la vida" , y "Si volviera a nacer, no cambiaría casi nada de mi vida". Estos ítems se responden siguiendo una escala de formato Likert con 7 opciones de respuesta que van desde $1=$ "En total desacuerdo" hasta $7=$ "Totalmente de acuerdo", suponiendo, mayores puntuaciones, una mayor satisfacción vital. El índice de consistencia interna para esta escala ha sido de $\alpha=, 72$.

El segundo cuestionario utilizado para evaluar el bienestar subjetivo es el Positive and Negative Affect Schedule (PANAS), de Watson, Clark y Tellegen (1988), validado en castellano por Sandín et al. (1999). Este instrumento contiene 20 ítems (10 por dimensión), con un formato de respuesta de $1=$ "Nunca" a 5 = "Casi siempre", suponiendo, por tanto, puntuaciones más elevadas un mayor afecto. La escala evalúa el afecto negativo (p. ej., nervioso, disgustado, atemorizado) y el afecto positivo (p.ej., entusiasmado, estimulado, motivado). El afecto negativo es una dimensión de malestar subjetivo que representa estados de ánimo aversivos, incluyendo hostilidad, malestar, nerviosismo, culpa y miedo. Por otro lado, el afecto positivo refleja niveles de energía, bienestar, entusiasmo y satisfacción. La estimación de su consistencia interna en este trabajo ha sido de $\alpha=, 72$ para el afecto positivo y de $\alpha=, 79$ para el afecto negativo.

\section{PROCEDIMIENTO}

El método de selección de la muestra en este estudio fue no probabilístico, por conveniencia, incluyendo a las personas participantes de acuerdo a la edad y el sexo. Para proceder con la fase de recogida de datos se solicitó el permiso correspondiente a las autoridades del Ministerio de Educación de la República Dominicana; luego se procedió a visitar los centros educativos participantes para 
informarles acerca de los objetivos del estudio y solicitar su autorización. A continuación, se convocó a las familias del alumnado a una reunión para exponerles el estudio y solicitar su consentimiento informado. Las pruebas se aplicaron dentro del horario lectivo y el tiempo de respuesta al conjunto de cuestionarios osciló entre 30 y 45 minutos. Las respuestas fueron completadas de forma anónima y voluntariamente.

\section{ANÁLISIS DE DATOS}

El análisis de los datos se realizó con el Software SPSS Statistics en su versión 23 para Windows. Para estimar la consistencia interna de los cuestionarios se utilizó el alfa de Cronbach y para estudiar la capacidad predictiva del apoyo social percibido sobre el bienestar subjetivo se llevó a cabo un análisis de regresión múltiple.

\section{RESULTADOS}

En la Tabla 1 se muestran los resultados obtenidos analizando el grupo de chicas. Los resultados obtenidos indican que, para las chicas, la familia sigue siendo una importante fuente de apoyo social a lo largo de toda la adolescencia. De hecho, es la fuente de apoyo que permite explicar en mayor medida las puntuaciones en satisfacción con la vida, de manera que cuanto mayor es el apoyo familiar percibido, mayor es la satisfacción vital que reportan las adolescentes, tanto las más jóvenes $(\beta=, 276 ; p=, 001)$, como las mayores $(\beta=, 301 ; p<, 001)$. Además, entre estas últimas, el apoyo de la familia permite explicar también menores puntuaciones en afecto negativo $(\beta=-, 264 ; p<, 001)$. La relevancia de las demás fuentes de apoyo, sin embargo, resulta distinta durante la adolescencia temprana y media. Así como

Tabla 1

Capacidad predictiva del apoyo social percibido sobre el bienestar subjetivo de las chicas ( $\mathrm{n}=579$ ).

\begin{tabular}{|c|c|c|c|c|c|c|c|c|c|c|c|c|c|}
\hline & & \multicolumn{4}{|c|}{ Satisfacción con la vida } & \multicolumn{4}{|c|}{ Afecto positivo } & \multicolumn{4}{|c|}{ Afecto negativo } \\
\hline & & $R^{2}$ & $\beta$ & $t$ & $p$ & $R^{2}$ & $\beta$ & $t$ & $p$ & $R^{2}$ & $\beta$ & $t$ & $p$ \\
\hline \multirow{4}{*}{ 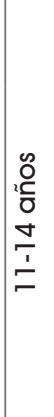 } & Constante & \multirow{4}{*}{.222} & - & 3.88 & $<.001$ & \multirow{4}{*}{.106} & - & 5.55 & $<.001$ & \multirow{4}{*}{.005} & - & 7.09 & $<.001$ \\
\hline & $\begin{array}{c}\text { Apoyo de } \\
\text { personas } \\
\text { significativas }\end{array}$ & & .227 & 2.67 & .008 & & .263 & 2.79 & .006 & & -.008 & -.082 & .935 \\
\hline & Apoyo familiar & & .276 & 3.42 & .001 & & .070 & .784 & .434 & & -.061 & -.660 & .510 \\
\hline & $\begin{array}{l}\text { Apoyo de } \\
\text { amistades }\end{array}$ & & .067 & .871 & .385 & & .038 & .443 & .659 & & -.011 & -.124 & .902 \\
\hline \multirow{4}{*}{ 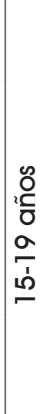 } & Constante & \multirow{4}{*}{.217} & - & .926 & $<.001$ & \multirow{4}{*}{.049} & - & 13.99 & $<.001$ & \multirow{4}{*}{.051} & - & 14.07 & $<.001$ \\
\hline & $\begin{array}{c}\text { Apoyo de } \\
\text { personas } \\
\text { significativas }\end{array}$ & & .086 & 1.22 & .225 & & .112 & 1.42 & .156 & & .091 & 1.13 & .259 \\
\hline & Apoyo familiar & & .301 & 4.99 & $<.001$ & & -.050 & -.746 & .456 & & -.264 & -3.89 & $<.001$ \\
\hline & $\begin{array}{l}\text { Apoyo de } \\
\text { amistades }\end{array}$ & & .160 & 2.34 & .020 & & .156 & 2.070 & .039 & & -.001 & -.009 & .993 \\
\hline
\end{tabular}


para las chicas más jóvenes el contar con el apoyo de una persona especial o significativa permite explicar mayores puntuaciones en su satisfacción vital $(\beta=, 227$; $p=, 008)$, y su afecto positivo $(\beta=, 263 ; p=, 006)$, entre las chicas de más edad es percibir el apoyo de las amistades lo que explica puntuaciones más elevadas tanto en satisfacción con la vida $(\beta=, 160 ; p=, 020)$ como en afecto positivo $(\beta=, 156 ; \beta=, 039)$.

Entre los chicos los resultados cambian ligeramente, observándose, en general, un menor peso del apoyo social sobre su bienestar subjetivo (Tabla 2). En el caso de los chicos, el percibir el apoyo de una persona especial o significativa es la única fuente que muestra capacidad explicativa sobre las puntuaciones del bienestar subjetivo tanto entre los chicos más jóvenes, como entre los mayores. Sin embargo, dicha capacidad predictiva difiere en cada grupo de edad.
Entre los chicos menores, percibir el apoyo de una persona significativa permite explicar mayores puntuaciones en satisfacción con la vida $(\beta=, 382 ; p=, 019)$, y en afecto positivo $(\beta=, 223 ; p=, 010)$, mientras que entre los chicos de mayor edad únicamente permite explicar menores puntuaciones en afecto negativo $(\beta=-, 198 ; p=, 023)$. En lo que respecta al apoyo familiar, al igual que ocurría entre las chicas, permite explicar mayores puntuaciones en satisfacción con la vida $(\beta=, 286 ; p<, 001)$, pero solo entre los chicos de más edad.

\section{DISCUSIÓN}

El objetivo general del presente estudio consistió en analizar la capacidad predictiva del apoyo social percibido sobre el bienestar subjetivo. En tal sentido, la primera hipótesis planteada se confirma parcialmente, ya

Tabla 2

Capacidad predictiva del apoyo social percibido sobre el bienestar subjetivo de los chicos $(n=451)$.

\begin{tabular}{|c|c|c|c|c|c|c|c|c|c|c|c|c|c|}
\hline & & \multicolumn{4}{|c|}{ Satisfacción con la vida } & \multicolumn{4}{|c|}{ Afecto positivo } & \multicolumn{4}{|c|}{ Afecto negativo } \\
\hline & & $R^{2}$ & $\beta$ & $t$ & $p$ & $R^{2}$ & $\beta$ & $t$ & $p$ & $R^{2}$ & $\beta$ & $t$ & $p$ \\
\hline \multirow{4}{*}{ 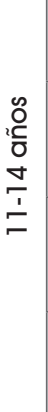 } & Constante & \multirow{4}{*}{.118} & - & 5.81 & $<.001$ & \multirow{4}{*}{.047} & - & 10.85 & $<.001$ & \multirow{4}{*}{.012} & - & 7.51 & $<.001$ \\
\hline & $\begin{array}{c}\text { Apoyo de } \\
\text { personas } \\
\text { significativas }\end{array}$ & & .382 & 2.40 & .019 & & .223 & 2.58 & .010 & & -.136 & -.811 & .419 \\
\hline & Apoyo familiar & & -.017 & -.110 & .912 & & .175 & 1.09 & .279 & & -.128 & -.808 & .421 \\
\hline & $\begin{array}{l}\text { Apoyo de } \\
\text { amistades }\end{array}$ & & -.046 & -.369 & .713 & & .089 & .678 & .499 & & .053 & .399 & .691 \\
\hline \multirow{4}{*}{ 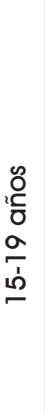 } & Constante & \multirow{4}{*}{.108} & - & 10.82 & $<.001$ & \multirow{4}{*}{.017} & - & 13.88 & $<.001$ & \multirow{4}{*}{.040} & - & 13.60 & $<.001$ \\
\hline & $\begin{array}{c}\text { Apoyo de } \\
\text { personas } \\
\text { significativas }\end{array}$ & & .105 & 1.28 & .203 & & .099 & 1.13 & .258 & & -.198 & -2.28 & .023 \\
\hline & Apoyo familiar & & .286 & 3.68 & $<.001$ & & .053 & .642 & .522 & & -.059 & -.784 & .434 \\
\hline & $\begin{array}{l}\text { Apoyo de } \\
\text { amistades }\end{array}$ & & -.067 & -.909 & .364 & & .024 & .306 & .760 & & -.073 & -.937 & .350 \\
\hline
\end{tabular}


que el apoyo social percibido ha mostrado capacidad predictiva sobre el bienestar subjetivo de adolescentes de la República Dominicana. Teniendo en cuenta que existen muy pocos estudios con población latinoamericana y ninguno con muestra de la República Dominicana, este trabajo puede aportar información interesante.

El primer objetivo específico ha sido analizar la capacidad predictiva de tres fuentes del apoyo social percibido (de la familia, de las amistades y de personas significativas) sobre los componentes del bienestar subjetivo (satisfacción con la vida, afecto negativo y afecto positivo). Los resultados muestran que el apoyo familiar predice la satisfacción con la vida y el afecto negativo; mientras el apoyo de las amistades y de personas significativas predice la satisfacción con la vida y el afecto positivo en el caso de las chicas. El apoyo familiar predice la satisfacción con la vida; y el apoyo de personas significativas predice la satisfacción con la vida, afecto positivo y afecto negativo entre los chicos.

El segundo objetivo específico de este estudio ha sido analizar la capacidad predictiva de las tres fuentes del apoyo social percibido (de la familia, de las amistades y de personas significativas) sobre los componentes del bienestar subjetivo (satisfacción con la vida, afecto negativo y afecto positivo), considerando el sexo de las personas participantes. En este sentido, puede considerarse confirmada la segunda hipótesis, en la que se esperaba distinta capacidad predictiva sobre los tres componentes del bienestar subjetivo en función del sexo. Se observa, en el caso de las chicas, una mayor importancia del apoyo familiar, mientras que en el caso de los chicos el apoyo percibido de personas significativas parece tener más relevancia sobre su bienestar subjetivo.

El tercer objetivo específico ha consistido en analizar la capacidad predictiva de las tres fuentes del apoyo social percibido (de la familia, de las amistades y de personas significativas) sobre los componentes del bienestar subjetivo (satisfacción con la vida, afecto negativo y afecto positivo) en función de la edad (adolescencia temprana y media). A este respecto se ha observado que la capacidad predictiva de las fuentes de apoyo social es distinta según la edad estudiada, confirmándose en parte, también, la tercera hipótesis planteada en el estudio. Concretamente, se ha constatado mayor peso de la familia entre las adolescentes mayores (en su satisfacción vital y afecto negativo), mientras que entre las jóvenes no llega a explicar su afecto. Al mismo tiempo, parece que entre las chicas jóvenes cobra más relevancia el apoyo de personas significativas para su satisfacción y afecto positivo, cumpliendo este papel las amistades entre las chicas mayores. En este estudio el apoyo familiar también resulta más relevante entre los chicos mayores, y se observa que, en función de la edad, la capacidad predictiva de las personas significativas cambia entre los chicos, siendo más relevante entre los más jóvenes, prediciendo su satisfacción con la vida y afecto positivo, y limitándose a menor afecto negativo entre los chicos de más edad.

En línea con investigaciones previas, los resultados muestran que la familia sigue siendo importante en la adolescencia, pues es la fuente de apoyo que permite explicar en mayor medida la satisfacción con la vida (Gutiérrez y Goncalves, 2013; RodríguezFernández et al., 2016) tanto de las chicas, en la adolescencia temprana y media, como de los chicos (adolescencia media). Estos resultados concuerdan, en parte, con los estudios que observaban un mayor peso del apoyo familiar frente al de las amistades a la hora de explicar la satisfacción con la vida (González-Carrasco et al., 2017; Sarriera et al., 2015; Rodríguez-Fernández et al., 2012), si bien dicha importancia parece ser mayor, en este trabajo, en el caso de las chicas. De hecho, es entre ellas (concretamente entre las mayores), entre quienes percibir mayor apoyo de la familia también explica menores puntuaciones de afecto negativo, algo observado previamente entre chicos y chicas adolescentes (Rodríguez-Fernández et al., 2016) y con estudiantes de Universidad (Brannan et al., 2013). Es posible que el hecho de que el apoyo de la familia tome especial relevancia en el bienestar y afecto positivo 
de los y las adolescentes de más edad, y especialmente entre las mujeres, se deba, en parte, a la evolución que los conflictos tienen a lo largo de la adolescencia. De hecho, se observa que la conflictividad familiar, más frecuente en la adolescencia temprana (Gaete, 2015) se reduce ligeramente con el transcurso de los años, y especialmente hacia el final de la adolescencia media (Iglesias, 2013). Además, las chicas tienden a mostrar menores conflictos, en general, y mayor comunicación familiar (Parra y Oliva, 2002), lo que podría favorecer el que estas acudan más a sus progenitores en busca de ayuda para la gestión de las emociones y afectos negativos.

Por otro lado, el apoyo de las amistades parece tener un peso más relevante en el bienestar subjetivo (satisfacción con la vida y afecto positivo) entre las chicas mayores que entre las jóvenes, sin mostrar capacidad explicativa significativa entre los chicos, en línea con los estudios que indican que las chicas confieren mayor importancia a sus relaciones de amistad (Barcelata et al., 2013; Barra, 2012).

En cuanto al apoyo de personas significativas, al igual que en otros estudios (Gallagher y Vella-Brodrick, 2008), permite explicar mayores puntuaciones en satisfacción con la vida y afecto positivo en la adolescencia temprana en ambos sexos, además de explicar menores puntuaciones en afecto negativo entre los chicos de más edad. No obstante, es difícil contrastar estos resultados con la investigación previa, ya que la mayor parte de los estudios se han realizado con muestra universitaria o adulta y no analizan posibles diferencias en función del sexo (Gallagher y Vella-Brodrick, 2008; Gülaçti, 2010; Matsuda et al., 2014). Puede que una explicación de estos resultados sea el inicio de las relaciones de pareja que ocurre en la adolescencia (Vongsirimas et al., 2018), y que suelen cobrar especial relevancia hacia el final de la adolescencia temprana (Navarro et al., 2017). También podría deberse al hecho de que, precisamente la adolescencia temprana tiende a identificarse como una época de fuerte involucramiento y necesidad de amistades exclusivas (Gaete, 2015), lo que podría traducirse en la importancia para el bienestar adolescente de contar con ese "mejor amigo íntimo" o esa "mejor amiga íntima". Además, otros estudios han observado similares resultados en cuanto al menor peso de las amistades entre los chicos, explicando que quizás ese menor apoyo sea sustituido por el de la pareja (Barcelata et al., 2013) o, en este caso, persona significativa, lo que explicaría la relevancia observada de dicha fuente en la adolescencia media de los chicos de este estudio. Entre las chicas, sin embargo, la mayor intimidad y proximidad con las amistades (Barcelata et al., 2013; Barra, 2012), que aumenta, además, con la edad (Gaete, 2015), podría explicar que la persona significativa no sea tan relevante en un contexto en el que el grado de intimidad con sus amistades, en general, es elevado. No obstante, es difícil confirmar estas explicaciones porque la interpretación de la "persona íntima" queda a juicio de quien responde, no pudiendo conocer con seguridad a quién hace referencia.

La menor capacidad explicativa del apoyo familiar y la nula capacidad explicativa del apoyo de amistades entre los chicos, que difiere de lo observado en estudios previos (Azpiazu et al., 2016), podría deberse a características específicas de cada muestra no tenidas en cuenta en este estudio, así como a la necesidad adolescente de mostrar independencia y autonomía en la resolución de sus problemas (Morgan y Roberts, 2010), algo que, además, se espera socialmente más de los hombres que de las mujeres, a quienes se ve como más dependientes (McLean y Anderson, 2009). También concuerda con la idea de que en la adolescencia las amistades masculinas son de menos calidad y menos íntimas, posiblemente por la socialización en la importancia y mantenimiento de las relaciones que reciben las mujeres, al contrario de lo esperado para el rol masculino (Leal, Ramos, Moreno y Rivera, 2013).

Este estudio presenta algunas limitaciones, ya que la capacidad predictiva del apoyo social en el bienestar subjetivo de la muestra analizada deja una amplia proporción de la varianza por explicar, por lo que sería interesante, en futuros estudios, considerar 
otras variables, tanto personales como de apoyo social (profesorado, apoyo formal, etc.) que puedan contribuir a conocer qué factores se encuentran implicados en el bienestar adolescente. Además, sería interesante utilizar análisis de ecuaciones estructurales, lo que permitiría aumentar el nivel de complejidad de estudio y proponer modelos teóricos explicativos de la variabilidad en los componentes del bienestar subjetivo. De igual forma, resultaría conveniente realizar estudios longitudinales que permitan analizar si el comportamiento de estas variables durante toda la etapa del desarrollo de la adolescencia concuerda con lo observado en este estudio transversal.

A pesar de las limitaciones mencionadas, esta investigación aporta información empírica relevante acerca de la capacidad predictiva del apoyo social en estudiantes de educación secundaria. En este sentido, los resultados de este estudio podrían servir como base para el desarrollo de programas de intervención cuyo objetivo sea mejorar las relaciones entre las diferentes fuentes de apoyo adolescente, para así poder contribuir en el mantenimiento y optimización del bienestar subjetivo durante la adolescencia, periodo vital complicado en el que puede verse comprometido por los numerosos cambios y retos a los que se ven sometidos los y las jóvenes.

\section{- Conflicto de intereses.}

Los autores declaran no tener ningún conflicto de intereses.

\section{- Agradecimientos}

Este trabajo ha recibido financiación del Ministerio de Educación Superior, Ciencia y Tecnología (MESCyT), República Dominicana. Por otra parte, se enmarca dentro de parte del trabajo realizado en el Grupo Consolidado de Investigación del Sistema Universitario Vasco IT934-16, así como del proyecto de investigación PPG17/61 de la Universidad del País Vasco y del proyecto EDU2017-83949-P del subprograma estatal de Generación del Conocimiento del Ministerio de Economía, Industria y Competitividad.

\section{REFERENCIAS}

Albuquerque, I., de Lima, M., Matos, M., \& Figueiredo, C. (2012). Personality and subjective well-being: What hides behind global analyses? Social Indicators Research, 105(3), 447-460. doi: 10.1007/s 11205 010-9780-7
Alfaro, J., Casas, F., \& López, V. (2015). Bienestar en la infancia y adolescencia. Psicoperspectivas, 14(1), 1-5.

Arechabala, M. C., \& Miranda, C. (2002). Validación de una escala de apoyo social percibido en un grupo de adultos mayores adscritos a un programa de hipertensión de la región metropolitana. Ciencia y Enfermería, 8(1), 49-55.

Atienza, F. L., Pons, D., Balaguer, I., \& García-Merita, M. (2000). Propiedades psicométricas de la escala de satisfacción con la vida en adolescentes. Psicothema, 12(2), 314-319.

Azpiazu, L., Esnaola, I., \& Sarasa, M. (2016). Apoyo social y bienestar subjetivo en la adolescencia. En J. L. Castejón (Coord.), Psicología y Educación: Presente y Futuro (pp. 230-243). Alicante: Asociación Científica de Psicología y Educación.

Balluerka, N., Gorostiaga, A., Alonso-Arbiol, I., \& Aritzeta, A. (2016). Peer attachment and class emotional intelligence as predictors of adolescents' psychological well-being: A multilevel approach. Journal of Adolescence, 53, 1-9. doi: 10.1016/i. adolescence.2016.08.009

Barcelata, B. E. E., Granados, A. M., \& Ramírez, A. F. (2013). Correlatos entre funcionamiento familiar y apoyo social percibido en escolares en riesgo psicosocial. Revista Mexicana de Orientación Educativa, 10(24), 65-70.

Barra, E. A. (2004). Apoyo social, estrés y salud. Psicología y Salud, 14, 237-243. doi: 10.25009/pys.v14i2.848

Barra, E. A. (2012). Influencia de la autoestima y del apoyo social percibido sobre el bienestar psicológico de estudiantes universitarios chilenos. Diversitas: Perspectivas en Psicología, 8(1), 29-38.

Bisquerra, A. R. (2010). Educación emocional y bienestar. Madrid: Wonters Kluwer.

Bowen, K. S., Uchino, B. N., Birmingham, W., Carlisle, M., Smith, T. W., \& Light, K. C. (2014). The stress-buffering effects of functional social support on ambulatory blood pressure. Health Psychology, 33(11), 1440-1443. doi: 10.1037/hea0000005 Bradburn, N. (1969). The structure of psychological well-being. Chicago: Aldine Publisling Company. 
Brannan, D., Biswas-Diener, R., Mohr, C. D., Mortazavi, S., \& Stein, N. (2013). Friends and family: A cross-cultural investigation of social support and subjective well-being among college students. The Journal of Positive Psychology, 8(1), 65-75. doi: 10.1080/17439760.2012.743573

Chen, W., Zhang, D., Pan, Y., Hu, T., Liu, G., \& Luo, S. (2017). Perceived social support and self-esteem as mediators of the relationship between parental attachment and life satisfaction among Chinese adolescents. Personality and Individual Differences, 108, 98-102. doi: 10.1016/i. paid.2016.12.009

Cunsolo, S. (2017). Subjective wellbeing during adolescence: A literature review on key factors relating to adolescent's subjective wellbeing and education outcomes. Studi Sulla Formazione, 20(1), 81-94. doi: 10.13128/Studi Formaz-20941

Dambi, J. M., Corten, L., Chiwaridzo, M., Jack, H., Mlambo, T., \& Jelsma, J. (2018). A systematic review of the psychometric properties of the cross-cultural translations and adaptations of the Multidimensional Perceived Social Support Scale (MSPSS). Health and Quality of Life Outcomes, 16(1), 2-19. doi: 10.1186/s12955-0180912-0

Diener, E. (1984). Subjective well-being. Psychological Bulletin, 95(3), 542-575. doi: 10.1037/0033-2909.95.3.542

Diener, E. (2009). Subjective well-being. En E. Diener (Ed.), In the science of well-being (pp. 11-58). New York, NY, US: Springer Science + Business Media.

Diener, E., Emmons, R. A., Larsen, R. J., \& Griffin, S. (1985). The satisfaction with life scale. Journal of Personality Assessment, 49(1), 71-75. doi: 10.1207/ s15327752ipa4901 13

Diener, E., Suh, E., Lucas, R., \& Smith, H. (1999). Subjective well-being: Tree decades of progress. Psychological Bulletin, 125(2), 276-302.

Fuentes, M. C., García, F., Gracia, E., \& Alarcón, A. (2015). Los estilos parentales de socialización y el ajuste psicológico. Un estudio con adolescentes españoles. Revista de Psicodidáctica, 20(1), 117-138. doi: 10.1387/RevPsicodidact. 10876

Gaete, V. (2015). Desarrollo psicosocial del adolescente. Revista Chilena de Pediatría, $86(6)$, 436-443. doi: 10.1016/i. rchipe.2015.07.005

Gallagher, E. N., \& Vella-Brodrick, D. A. (2008). Social support and emotional intelligence as predictors for subjective well-being. Personality and Individual Differences, 44, 1551-1561. doi: 10.1016/i.paid.2008.01.011

González, E., Martínez, V., Molina, T., George, M., Sepúlveda, R., Molina, R., \& Hidalgo-Rasmussen, C. (2016). Diferencias de género en la calidad de vida relacionada con la salud en adolescentes escolarizados chilenos. Revista Médica de Chile, 144(3), 298-306. doi: 10.4067/ S0034-98872016000300004

González, S. L., Gaxiola, J. C. R., \& Venezuela, E. R. H. (2018). Apoyo social y resiliencia: predictores de bienestar psicológico en adolescentes con suceso de vida estresante. Psicología y Salud, 28(2), 167-176.

González, N. I., Torres, M. A., \& Morelaro, G. S. (2020). Apoyo Social, Autoestima y Bienestar Subjetivo en Escolares. El caso de México y Argentina. Acta de Investigación Psicológica, 10(2), 65-79.

González-Carrasco, M., Casas, F., Viñas, F., Malo, S., Gras, M. E., \& Bedin, L. (2017). What leads subjective well-being to change throughout adolescence? An exploration of potential factors. Child Indicators Research, 10(1), 33-56.

Gülaçti, F. (2010). The effect of perceived social support on subjective well-being. Procedia Social and Behavioral Sciences, 2, 3844-3849.

Gutiérrez, M., \& Gonçalvez, T. O. (2013). Activos para el desarrollo, ajuste escolar y bienestar subjetivo de los adolescentes. International Journal of Psychology and Psychological Therapy, 13(3), 339-355.

Haanpää, L., Kuula, M., \& Hakovirta, M. (2019). Social relationships, child poverty, and children's life satisfaction. Social Sciences, 8(2), 1-13. doi: 10.3390/ socsci8020035

Hernando, Á., Oliva, A., \& Pertegal, M. Á. (2013). Diferencias de género en los estilos 
de vida de los adolescentes. Psychosocial Intervention, 22(1), 15-23.

Iglesias, J. D. (2013). Desarrollo del adolescente: aspectos físicos, psicológicos y sociales. Pediatría Integral, 17(2), 88-93.

Kong, F., Zhao, J., \& You, X. (2012). Emotional intelligence and life satisfaction in Chinese university students: The mediating role of self-esteem and social support. Personality and Individual Differences, 53(8), 10391043. doi: 10.1016/i.paid.2012.07.032

Leal, E., Ramos, P., Moreno, M. C. R., \& Rivera, F. (2013). Características de las relaciones de amistad durante la adolescencia: diferencias entre chicos y chicas en España. Revista de Psicologia da Criança e do Adolescente, 3(2), 315-327.

Lin, N. (1986). Conceptualizing social support. En N. Lin, A. Dean, y W. Ensel (Eds.), Social support, life events, and depression (pp. 17-30). New York: Academic Press.

Liu, W., Li, Z., Ling, Y., \& Cai, T. (2016). Core self-evaluations and coping styles as mediators between social support and well-being. Personality and Individual Differences, 88, 35-39. doi: 10.1016/i. paid.2015.08.044

Matsuda, T., Tsuda, A., Kim, E., \& Deng, K. (2014). Association between perceived social support and subjective well-being among Japanese, Chinese, and Korean college students. Psychology, 5(6), 491 499. doi: 10.4236/psych.2014.56059

McLean, C. P., \& Anderson, E. R. (2009). Brave men and timid women? A review of the gender differences in fear and anxiety. Clinical Psychology Review, 29(6), 496505. doi: 10.1016/i.cpr.2009.05.003

Morgan, J. P., \& Roberts, J. E. (2010). Helping bereaved children and adolescents: Strategies and implication for counselors. Journal of Mental Health Counseling, 32(3), 206-217. doi: 10.17744/ mehc.32.3.nu $2 \mathrm{kx} 6267 \mathrm{~g} 81 \mathrm{~m} 81 \mathrm{w}$

Navarro, D., Monteserrat C., Malo, S., González, M., Casas, F., \& Crous, G. (2017). Subjective well-being: what do adolescents say? Child \& Family Social Work, 22(1), 175-184.

Navarro-Loli, J. S., Merino-Soto, C., Domínguez-Lara, S., \& Lourenço, A. (2019) Estructura interna de la Multidimensional
Scale of Perceived Social Support (MSPSS) en adolescentes peruanos. Revista Argentina de Ciencias del Comportamiento, $17(1), 38-47$.

Norris, S. M., \& Ayres, C. G. (2016). Factors influencing the health promoting physical activity behaviors of diverse urban adolescents. Journal of Nursing Practice Applications and Reviews of Research, 6(1), 16-23. doi: 10.2105/AJPH.2014.302481 Oberle, E., Schonert-Reichl, K. A., \& Zumbo, B. D. (2011). Life satisfaction in early adolescence: Personal, neighbourhood, school, family, and peer influences. Journal of Youth and Adolescence, 40(7), 889901. doi: 10.1007/s10964-010-9599-1

Orcarsita, L. T., \& Uribe, A. F. (2012). La importancia del apoyo social en el bienestar de los adolescentes. Psychologia. Avances de la Disciplina, 4(2), 62-82.

Palomar-Lever, J., \& Victorio-Estrada, A. (2014). Determinants of subjective wellbeing in adolescent children of recipients of the Oportunidades human development program in Mexico. Social Indicators Research, 118(1), 103-124.

Parra, Á., \& Oliva, A. (2002). Comunicación y conflicto familiar durante la adolescencia. Anales de Psicología, 18(2), 215-23.

Prabhu, S. G., \& Shekhar, R. (2017). Resilience and perceived social support among school-going adolescents in Mangaluru. Indian Journal of Social Psychiatry, 33(4), 359-364. doi: 10.4103/iisp.ijsp 10816 Rodríguez-Fernández, A., Droguett, L., \& Revuelta, L. (2012). Ajuste escolar y personal en la adolescencia: El papel del autoconcepto académico y del apoyo social percibido. Revista de Psicodidáctica, 17(2), 397-413. doi: 10.1387/Rev. Psicodidact.3002

Rodríguez-Fernández, A., \& GoñiGrandmontagne, A. (2011). La estructura tridimensional del bienestar subjetivo. Anales de Psicología, 27(2), 327-332.

Rodríguez-Fernández, A., Ramos-Díaz, E., Ros, I., Fernández-Zabala, A., \& Revuelta, L. (2016). Bienestar subjetivo en la adolescencia: el papel de la resiliencia, el autoconcepto y el apoyo social percibido. Suma Psicológica, 2(3), 60-69. doi: $\underline{10.1016 / i . s u m p s i .2016 .02 .00}$ 
Ronen, T., Hamama, L., Rosenbaum, M., \& Mishely-Yarlap, A. (2016). Subjective well-being in adolescence: The role of self-control, social support, age, gender, and familial crisis. Journal of Happiness Studies, 17(1), 81-104.

Sandín, B., Chorot, P., Lostao, L., Joiner, T. E., Santed, M. A., \& Valiente, R. M. (1999). Escalas PANAS de afecto positivo y negativo: validación factorial y convergencia transcultural. Psicothema, $17(1), 37-51$.

Sarriera, J. C, Bedin, L., Abs, D., Calza, T., \& Casas, F. (2015). Relationship between social support, life satisfaction and subjective well-being in Brazilian adolescents. Universitas Psychologica, 14(2), 459-474. doi: 10.11144/ Javeriana.upsy 14-2.rbss

Trejos-Herrera, A. M., Bahamón, M. J., Alarcón-Vásquez, Y., Vélez, J. I., \& Vinaccia, S. (2018). Validity and reliability of the multidimensional scale of perceived social support in Colombian adolescents. Psychosocial Intervention, 27(1), 56-63. doi: 10.5093/pi2018al

Vongsirimas, N., Phetrasuwan, S., Thanoi, W., \& Yobas, P. K. (2018). Psychometric properties of the Multi-dimensional Scale of Perceived Social Support among Thai youth. Thai Pharmaceutical and Health Science Journal, 13(3), 135-141.
Wang, Y. F., \& Zauszniewski, J. A. (2018). Predictors of resourcefulness in preadolescent children. Western Journal of Nursing Research, 40(8), 1163-1183. doi: $10.1177 / 0193945917700139$

Watson, D., Clark, L. A., \& Tellegen, A. (1988). Development and validation of brief measures of positive and negative affect: the PANAS scales. Journal of Personality and Social Psychology, 54(6), 1063-1070.

Yang, C., Xia, M., Han, M., \& Liang, Y. (2018). Social support and resilience as mediators between stress and life satisfaction among people with substance use disorder in China. Frontiers in Psychiatry, 9, 1-7. doi: 10.3389/fpsyt.2018.00436

Zavala, M. A., Valadez, M. D., \& Vargas, M. C. (2008). Emotional intelligence and social skills in adolescents with high social acceptance. Electronic Journal of Research in Educational Psychology, 6(2), 319-338.

Zeidner, M., Matthews, G., \& Shemesh, D. O. (2016). Cognitive-social sources of wellbeing: differentiating the roles of coping style, social support and emotional intelligence. Journal Happiness Studies, 17, 2481-2501.

Zimet, G. D., Dahlem, N. W., Zimet, S. G., \& Farley, G. K. (1988). The multidimensional scale of perceived social support. Journal of Personality Assessment, 52, 30-41. 